\title{
Certificación orgánica: un intangible que debe ser reconocido en los estados financieros*
}

\author{
Yaquelin Castañeda Novoa \\ Jenny Carolina Gómez Elizalde*
}

Recibido: 10 de octubre de 2012

Aprobado: 14 de noviembre 2012

Castañeda, Y. y Gómez, J. (2012). Certificación orgánica: un intangible que debe ser reconocido en los estados financieros. Activos, 19, 183-197

\section{JEL: M 49}

\section{Resumen}

El propósito de este documento es presentar el concepto de certificación orgánica y contextualizar su proceso de obtención en Colombia, con la finalidad de indicar las características que permiten su reconocimiento contable como un activo intangible. De este modo, se hace una comparación del concepto de certificación orgánica establecido por el Ministerio de Agricultura y Desarrollo Rural, y los conceptos de activo e intangible estipulados en la norma nacional e internacional.

\footnotetext{
${ }^{*}$ El documento corresponde al que fue presentado al VII Congreso de Estudiantes de Contaduría Pública de la Universidad Santo Tomás. Ha sido desarrollado en el marco del semillero de investigación Sinetco, de la Facultad de Contaduría Pública (tipo IV).

** Estudiantes del programa de Contaduría Pública de la Universidad Santo Tomás. Correos electrónicos: jackelinecastañeda@usantotomas.edu.co y yennygomez@ usantotomas.edu.co
} 
A partir de esto, se concluye que la certificación orgánica debería ser reconocida contablemente como un intangible, debido a que esta cumple con las características esenciales de un activo: beneficio económico futuro, recurso controlado y valoración de costos.

El documento plantea una serie de cuestiones que permiten avanzar en nuevos problemas de investigación, alrededor de los problemas que tiene para las organizaciones la gestión de los activos intangibles su no reconocimiento, y en especial en el marco de una dinámica donde lo ambiental se vuelve cada vez más importante se convierte en un problema de productividad y competitividad enorme.

\title{
Palabras clave:
}

certificación orgánica, activo ambiental, activos intangibles, gestión ambiental, información ambiental.

Castañeda, Y. y Gómez, J. (2012). Organic certification: an intangible that should be recognized in the financial statements. Activos, 19, 183-197

\begin{abstract}
The purpose of this paper is to present the concept of organic certification and contextualize the process to obtain it in Colombia, in order to indicate the characteristics that allow its accounting recognition as an intangible asset. Thus, a comparison is made of the concept of organic certification established by the Ministry of Agriculture and Rural Development, and the concepts of asset and intangible stipulated in the national and international standard.
\end{abstract}

From this, it is concluded that organic certification should be recognized accounting-wise as an intangible, since it meets the essential 
characteristics of an asset: future economic benefit, controlled resource and cost assessment.

The paper raises a number of issues that allow progressing on new research problems, surrounding the problems that present for organizations the management of intangible assets their non-recognition, particularly in the context of a dynamic framework where the environment is becoming increasingly more important it becomes a problem of enormous productivity and competitiveness.

\section{Keywords:}

organic certification, environmental asset, intangible assets, environmental management, environmental information.

Castañeda, Y. y Gómez, J. (2012). La certification biologique: un intangible qui doit être reconnu dans les états financiers. Activos, 19, 183-197

\section{Résumé}

Le but de ce document est de présenter le concept de certification biologique et contextualiser son processus dobtention en Colombie, dans le but d'indiquer les caractéristiques qui permettent sa reconnaissance comptable comme actif intangible. De cette façon, on fait une comparaison du concept de certification biologique établit par le Ministère de l'Agriculture et le Développement Rural et des concepts d'actif et intangible de la norme nationale et internationale.

À partir de cela, on peut conclure que la certification biologique devrait être reconnue dans la comptabilité comme un intangible, du fait que celle-ci respecte les caractéristiques essentielles d'un actif : avantage économique futur, contrôle des ressources et évaluation des coûts. 
Le document propose une série de questions qui permettent d'avancer dans de nouveaux problèmes de recherche, autour des problèmes qu'a, pour les organisations, la gestion des actifs immatériels, leur manque de reconnaissance, et spécialement dans le cadre d'une dynamique dans laquelle l'environnemental devient de plus en plus important chaque fois devient un problème de productivité et de compétitivité énorme.

\section{Mots-clés:}

certification biologique, actif environnemental, actifs intangibles, gestion environnementale, information environnementale.

\section{Introducción}

En los últimos años se ha incrementado la preferencia por consumir productos orgánicos, coincidente con la tendencia mundial de una alimentación sana. Esta creciente demanda representa para las empresas una oportunidad de incrementar sus ingresos. La certificación orgánica busca asegurar al consumidor el origen natural de los productos que adquiere. De acuerdo con lo anterior y teniendo en cuenta el impacto que conlleva adquirir para una empresa dicha certificación, surge desde la profesión contable la necesidad de analizar sus características y determinar la pertinencia de su revelación y representación en los estados financieros de las empresas. Frente a este panorama, el presente documento tiene como objetivo general indicar las características de las certificaciones orgánicas que permiten su reconocimiento contable como un activo intangible. Desde esta consideración, es necesario presentar el concepto de certificación orgánica y contextualizar su proceso de obtención, en este caso para las empresas colombianas. Para ello, el documento se subdivide en tres secciones: en primer lugar, se contextualiza el concepto de certificación orgánica y se especifica el procedimiento por seguir para las empresas colombianas que desean adoptar la normativa de producción orgánica. Posteriormente, se analizan los conceptos de activo intangible estipulados en la normativa nacional 
e internacional, con el fin de establecer las características que permitan argumentar que la certificación orgánica es susceptible de ser reconocida como un activo intangible. Finalmente, se plantean las conclusiones que presentan de manera resumida los aspectos desarrollados en el documento producto de la investigación realizada.

\section{Generalidades de la certificación orgánica}

Para contextualizar el concepto de certificación orgánica, es importante resaltar el papel de la agricultura en la evolución del hombre. Desde su origen, el ser humano ha buscado la forma de suplir sus necesidades, realizando prácticas como la recolección, la caza, la pesca, y posteriormente la agricultura, como solución inicial a la escasez de alimentos. En los años cincuenta, como consecuencia del crecimiento demográfico, el científico Norman Bourlaug dio inicio a la Revolución Verde, un conjunto de tecnologías integradas por componentes materiales, que tenían como objetivo mitigar el hambre en el mundo y conseguir una mayor producción en las cosechas (Pons, 2011; Organización de las Naciones Unidas para la Alimentación y la Agricultura [FAO], 1996).

La utilización de nuevas técnicas en tierras aptas para el cultivo y en entornos socioeconómicos propicios tuvo como resultado un gran aumento de los rendimientos y los ingresos para muchos agricultores de Asia y de algunos países en desarrollo de otros continentes (FAO, 1996). No obstante, dicho proceso falló, pues su propósito de mitigar el hambre y sus efectos fueron contraproducentes. Autores como Pat Mooney y Cary Fowler (citados en Ruiz, 2011), ambos ganadores del Nobel Alternativo en 1985, argumentaban "que el problema no era simplemente la escasez de los alimentos y la solución no era solo el aumento de la producción”. A su vez, Helena Paul (citada en Ruiz, 2011, p. 16) planteaba:

que como resultado de la Revolución Verde, áreas enteras de investigación sobre fertilidad de suelos, cultivos mixtos, manejo de agua y otras prácticas 
sustentables, que posiblemente podrían duplicar los rendimientos, fueron pasadas por alto a medida que los científicos se enfocaban en encontrar la combinación genética perfecta, un enfoque con grandes limitaciones.

Dicho progreso tecnológico dio origen a lo que se conoce hoy en día como alimentos transgénicos. El término transgénico se descompone en trans (mover, trasladar) y génico (información genética). En efecto, un organismo transgénico o genéticamente modificado (OGM) es todo aquel que tenga incorporado un gen extraño. Pero extraño no quiere decir indeseable. Por el contrario, la transgénesis se utiliza en la agricultura para incorporar genes en los cultivos y las plantas, que aunque provienen de otros organismos, son considerados en principio deseables; es decir, genes que producen resistencia a plagas y enfermedades, rendimientos más altos, producciones de mejor calidad y contenido nutricional, y tolerancia al calor, al frío y a la sequía (Arias, 2007).

Lo anterior significa que la transgénesis fue vista a nivel mundial como un progreso positivo; sin embargo, en la medida en que se fue desarrollando, se detectaron grandes consecuencias consideradas como desventajas, que afectan tanto al ser humano como al medio ambiente. Dentro de dichas consecuencias se encuentran: el incremento del uso de tóxicos en la agricultura, la contaminación genética y del suelo, la pérdida de biodiversidad, el desarrollo de resistencias en insectos, los riesgos sanitarios y los efectos no deseados en otros organismos (Greenpeace, 2012).

Como oposición a la incorrecta e insostenible forma de producir y en especial de un incremento en el uso de pesticidas y fertilizantes químicos durante la Revolución Verde, surge la agroecología, definida como "la base científica de la agricultura ecológica" (Altieri, citado en Sevilla, 2004, p.13), conocida esta última "como la práctica que trabaja bajo el concepto de producción sostenible y competitividad, sin detrimento de los recursos naturales, en aras del crecimiento económico y del mejoramiento de la calidad de vida de la población" (Ministerio de Agricultura y Desarrollo Rural, 2007, p. 1). 
En un principio no era necesario asegurar la producción sostenible de las prácticas llevadas por la agricultura, debido al vínculo de suma confianza existente entre consumidor y productor. Esta relación se rompe al introducir prácticas que alteran la producción de alimentos, lo que conlleva la necesidad de garantizar al consumidor el origen del producto, certificando que los cultivos de donde proviene siguen las normas de producción orgánica (Soto, 2002). Los primeros estándares de la certificación orgánica fueron elaborados como códigos voluntarios de grupos de interés de productores o consumidores. Como respuesta a ello, en Europa y Estados Unidos se presenta la idea de inspecciones independientes, donde organizaciones como California Certified Organic Farmers (CCOF), Oregón Tilth (OTCO) y Organic Growers Buyer's Association (OGBA) desarrollaron normas o estándares para la producción orgánica (De la Rosa Santamaría, 2010).

En Colombia, según el Ministerio de Agricultura y Desarrollo Rural (MADR), la agricultura orgánica no solo permite mayor calidad de los productos, también hace que las tierras tengan un ciclo más largo y duradero. Gracias a estas ventajas, ha establecido el proceso para que los productos se consideren ecológicos y puedan ser comercializados en nuevos mercados. Como primer paso para entrar en el mundo de la producción ecológica se debe conocer y cumplir la resolución 187 de 2006 del MADR, en la cual se reglamenta la producción y comercialización de productos ecológicos y su proceso de certificación.

Esta normativa establece en el país unos lineamentos para la producción primaria, procesamiento, empacado, etiquetado, almacenamiento, certificación, importación, comercialización y el sistema de control de productos agropecuarios ecológicos. Dicha resolución define el término "sistema de producción ecológica”, pero en general los términos ecológico, orgánico o biológico son sinónimos y enmarcan todos los sistemas agrícolas que promueven la producción agropecuaria de manera sana y segura, desde el punto de vista ambiental, social y económico (Ministerio de Agricultura y Desarrollo Rural, s. f.). 
En términos generales, el procedimiento para conseguir la certificación ecológica de un producto, según el MADR se indica en la tabla 1.

Tabla 1. Proceso de certificación orgánica para el productor

\begin{tabular}{|l|l|}
\hline Paso 1: & $\begin{array}{l}\text { El productor que desea certificar sus productos debe identifi- } \\
\text { car las entidades certificadoras reconocidas en los mercados } \\
\text { en los cuales pretende comercializar sus productos. Dicha } \\
\text { Identificar las entida- } \\
\text { entidad debe estar autorizada (o en proceso de autorización) } \\
\text { por el Ministerio de Agricultura y Desarrollo Rural, y a su vez } \\
\text { debe estar acreditada ante la Superintendencia de Industria } \\
\text { y Comercio. }\end{array}$ \\
\hline $\begin{array}{l}\text { Paso 2: } \\
\text { Solicitar cotización e } \\
\text { anformación necesaria certificadora }\end{array}$ & $\begin{array}{l}\text { El productor debe solicitar una cotización y entrega de docu- } \\
\text { mentos de referencia a la entidad certificadora, con el fin de } \\
\text { aspectos específicos del proceso de certificación dependen de } \\
\text { la normativa que la entidad certificadora tome como referencia. }\end{array}$ \\
\hline $\begin{array}{l}\text { Paso 3: } \\
\text { Selección final de la } \\
\text { certificadora }\end{array}$ & $\begin{array}{l}\text { Teniendo en cuenta las condiciones ofrecidas por cada entidad } \\
\text { certificadora, el productor debe seleccionar aquella con la cual } \\
\text { desea firmar contrato e iniciar el proceso de certificación. }\end{array}$ \\
\hline $\begin{array}{l}\text { Paso 4: } \\
\text { Solicitar el Sello } \\
\text { Ecológico }\end{array}$ & $\begin{array}{l}\text { Una vez finalizado el proceso de certificación, el productor } \\
\text { tiene como opción solicitar el "Sello Único Nacional de } \\
\text { Alimento Ecológico" al MADR, el cual debe llevar el empaque } \\
\text { del producto, adjuntando a la solicitud el certificado ecológico } \\
\text { emitido por la certificadora. }\end{array}$ \\
\hline
\end{tabular}

Modificado de MADR (2007)

Dentro del proceso de certificación, se puede evidenciar que las certificadoras juegan un papel muy importante dentro de la evaluación y seguimiento de las empresas que cultivan productos orgánicos, dado que son quienes determinan si se cumple con las normas de producción orgánica. Para las empresas colombianas las certificadoras más conocidas son 
Corporación Colombia internacional (CCI), Biotropico, Cotecna, Biolatina, Ecocert- Colombia y Ceres-Colombia.

\section{La certificación orgánica como activo intangible}

Según el MADR (2007), la certificación orgánica es un término que indica que los productos se han obtenido de acuerdo con las normas de la producción ecológica, y que están respaldados por un organismo o entidad de certificación autorizada. De acuerdo con las características que abarca dicha definición, es relevante mencionar que por su naturaleza, la certificación orgánica es considerada un recurso inmaterial para la empresa que lo adquiere, respaldado por un documento escrito que genera confianza en las relaciones consumidor-productor de las transacciones comerciales nacionales e internacionales de productos orgánicos.

La certificación orgánica da al productor la oportunidad de incrementar sus ingresos a través del precio de venta y puede ser reconocida contablemente en las empresas certificadas como un derecho intangible, puesto que posee las características de un activo susceptible de ser presentado en los estados financieros.

Según el marco conceptual para la información financiera emitido por el IASB (International Accounting Standards Board):

Un activo es un recurso controlado por la entidad como resultado de sucesos pasados, del que la entidad espera obtener, en el futuro, beneficios económicos. Se reconoce un activo en el balance cuando es probable que se obtengan del mismo beneficios económicos futuros para la entidad, y además el activo tiene un costo o valor que puede ser medido con fiabilidad (p. A40-A41).

Por su parte, en Colombia el Decreto 2649 de 1993 define que: "Un activo es la representación financiera de un recurso obtenido por el ente 
económico como resultado de eventos pasados, de cuya utilización se espera que fluyan a la empresa beneficios económicos futuros" (p. 7).

Teniendo en cuenta los conceptos anteriores, se puede afirmar que la definición del marco conceptual hace énfasis en tres características esenciales de los activos: el beneficio económico futuro, el control de los recursos por la entidad y los costos incurridos para adquirirlos. El Marco Conceptual menciona que los beneficios económicos futuros incorporados a un activo permiten contribuir directa o indirectamente a los flujos de efectivo de la entidad. La NIC 38 "activos intangibles" especifica que una entidad controlará este tipo de activos, siempre y cuando tenga el poder de obtener beneficios económicos futuros que procedan del mismo, y define su costo como el importe de efectivo pagado, o su valor razonable, en el momento de su adquisición.

De la misma forma, el Decreto 2649 de 1993 indica como característica esencial de todos los activos el beneficio económico futuro; sin embargo, la definición de su costo se especifica en el concepto de activo intangible indicando que: "El valor o costo histórico de estos activos debe corresponder al monto de las erogaciones claramente identificables en que efectivamente se incurra o se deba incurrir para adquirirlos, formarlos o usarlos" (p. 14).

Desde este argumento, se afirma que la certificación orgánica es un activo intangible, puesto que cumple con las tres características esenciales mencionadas anteriormente. En cuanto al beneficio económico futuro, la certificación orgánica da al productor la oportunidad de establecer un mayor precio a los productos orgánicos en comparación con aquellos producidos de manera convencional. En Colombia existen aproximadamente 49000 hectáreas de producción orgánica certificadas, lo que representa el 0,005 $\%$ de la producción mundial; por ende, las empresas colombianas tienen la posibilidad de aprovechar la demanda creciente de los productos orgánicos a nivel mundial para diversificar sus mercados (Bedoya, 2011). Asimismo, la certificación orgánica permite alcanzar un beneficio económico a largo plazo y reducir costos a la empresa, ya que inicialmente el productor tendrá 
que incurrir en elevados costos para implementar procesos productivos que garanticen la calidad orgánica de los productos. Sin embargo, en el largo plazo, la eliminación de prácticas convencionales como el uso de pesticidas y fertilizantes químicos de alto precio permitirá ajustar los costos de producción y mantener e incluso incrementar el margen de utilidad de las empresas convencionales. Por otra parte, la certificación crea una imagen o ventaja competitiva a la compañía y propicia su acceso a un mercado más selecto a nivel nacional y la posibilidad de realizar actividades de exportación a países donde el carácter orgánico de los productos es requisito fundamental para las relaciones comerciales.

Por otra parte, la certificación orgánica es un recurso que implica dos tipos de control por parte de la entidad: antes de adquirirla, el control es indirecto ya que la empresa administra adecuadamente sus procesos productivos de forma tal que adopta la normativa establecida, _-por ejemplo, la indicada por el MADR en Colombia - y garantiza la obtención de la certificación. Luego, dicho control se vuelve directo y permanente, dado que la certificación requiere renovación anual por parte de la entidad encargada, a través de una inspección que suele ser realizada sin previo aviso. Este hecho obliga a la entidad a actualizar de forma constante sus procesos productivos, y adicional a ello a controlar los riesgos que puede ocasionar el hecho de perder la certificación por factores como el clima, el tiempo, la decisión de terceros sobre la zona productiva, entre otros.

Con relación al tema del costo, último elemento indicado en el concepto de activo intangible, la certificación orgánica implica una serie de erogaciones que deberían ser incluidas en el valor de adquisición o costo histórico de dicho activo. Estos valores incluyen de forma general conceptos como inscripción, inspección, capacitación y adquisición del sello que da garantía sobre la característica orgánica del producto. Cabe aclarar que estos conceptos pueden variar de acuerdo con la entidad que certifica los productos orgánicos; no obstante, el costo por capacitación suele ser el más elevado y relevante para adquirir la certificación, ya que requiere de un proceso de formación y de cultura que abarca, tanto la organización, como su entorno. 
Teniendo la noción del concepto de certificación orgánica y de su impacto para las empresas que la adquieren, es pertinente realizar una síntesis de lo desarrollado anteriormente. Inicialmente se identifica la certificación como un recurso inmaterial, que permite establecer un vinculo de confianza en las transacciones realizadas entre consumidor y productor; asimismo, se reconoce la posibilidad que tiene el productor de obtener un mayor beneficio y con base en ello se procede a relacionar las características que permiten reconocer la certificación orgánica como un activo intangible en los estados financieros, según la normativa nacional (Decreto 2649 de 1993) e internacional (marco conceptual para la información financiera y la NIC 38). Finalmente, se concluye que la certificación orgánica cumple con las tres características esenciales de un activo intangible: beneficio económico futuro, un mayor precio de los productos con relación a los convencionales, la posibilidad de reducir costos y la opción de acceder a mercados internacionales; recurso controlado por la empresa de forma directa e indirecta y costo, y todas las erogaciones que se incluyen en su valor de adquisición.

\section{Conclusiones}

En primer lugar, es necesario tener en cuenta que el ser humano, al cuestionar el origen de los productos que consume, crea conciencia de lo importante que es tener una alimentación sana y balanceada, razón por la cual los productos orgánicos caracterizados por ser de origen natural y con un alto nivel nutritivo toman un papel relevante en el mercado. De este modo, la certificación orgánica surge con el propósito de garantizar al consumidor que los productos que adquiere cumplen con la normativa que permite denominarlos como orgánicos.

Por otra parte, la certificación orgánica debería ser reconocida contablemente como un intangible, debido a que esta cumple con las características esenciales de un activo: beneficio económico futuro, recurso controlado y valoración de costos. En palabras de Cañibano (2000): 
el punto crucial para distinguir entre activo y gasto está en su vinculación o no con rendimientos futuros. Si se trata de costes incurridos para solucionar situaciones pasadas o de rendimientos difícilmente previsibles estaríamos ante gastos, mientras que si se trata de costes incurridos hoy que darán lugar a probables rendimientos futuros nos encontraríamos ante auténticos activos (p. 86).

De acuerdo con lo anterior, para obtener la certificación orgánica el productor incurre en una serie de erogaciones, que pueden ser activadas o reconocidas como costo del intangible, puesto que pueden ser recuperadas y dan lugar a beneficios económicos futuros, los cuales se ven reflejados al incrementar sus ingresos a través del precio de venta de sus productos y en la posibilidad de reducir y ajustar los elevados costos necesarios para adoptar un proceso sostenible.

Frente a este panorama, se plantean otros interrogantes que podrían ser abordados desde la disciplina contable, como por ejemplo: ¿es la certificación orgánica un activo intangible adquirido o formado?, ¿qué efectos tributarios reconocería la entidad certificadora si en su tratamiento contable reconoce la certificación como un activo intangible y no como un gasto?

\section{Referencias}

Arias, A. F. (2007). Transgénicos en Colombia. Ministerio de Agricultura $y$ desarrollo Rural. Recuperado de http://www.Minagricultura.gov.co/media/55836107.pdf

Bedoya, C. (2011). Colombia necesita más producción de orgánicos para exportar. Momento empresarial. Recuperado de http://www.Momentoempresarial. net/noticias/colombia-necesita-mas-produccion-de-organicos-para-exportar

Cañibano, L. (2000). El medio ambiente en la contabilidad de las empresas. Revista Legis del Contador, 2, 81-86.

Colombia, Ministerio de Agricultura y Desarrollo Rural (2007). La agricultura ecológica en Colombia. Ministerio de Agricultura y Desarrollo Rural. 
Recuperado de http://www.minagricultura.gov.co/archivos/articulo_de_agricultura_ecologica._madr._2007.pdf

Colombia, Ministerio de Agricultura y Desarrollo Rural (2012). Agricultura Limpia. Agricultura orgánica o ecológica. Ministerio de Agricultura y Desarrollo Rural. Recuperado de http://www.Minagricul tura.gov.co/archivos/guia_de_ agricultura_ecologica.pdf

De la Rosa, E. (2010) Regulación nacional y equivalencia internacional de productos orgánicos. Recuperado de http://www.Agriculturaecologica.com/ attachments/347REGULACION\%20Y\%20EQUIVALENCIAS.pdf

Greenpeace (2012). Guía roja y verde de alimentos transgénicos $5^{\mathrm{a}}$ edición. Greenpeace. Recuperado de http://www.Greenpeace.org/espana /Global/ espana/reporte/transgénicos/Textos-listado-5edicion.pdf

Organización de las Naciones Unidas para la Alimentación y la Agricultura (FAO) (noviembre, 1996). Cumbre mundial sobre la alimentación. Recuperado de http://www.fao.org/docrep/003/w2612s/w2612s06.htm

Pons, J. O. (2011, 2 de enero). Discurso de Norman Borlaug, el padre de la "Revolución Verde", con motivo de su investidura como Doctor "Honoris Causa”. Recuperado de http://constitucionweb.blogspot.Com/2011/01/ discurso-de-norman-borlaug-el-padre de_02.html

Ruiz, M. C. (2008, 8 de agosto). El gran debate de la agricultura mundial. Recuperado de http://www.ecoportal.net/Temas_ Especiales/Desarrollo_ Sustentable/El_gran_debate_de_la_agricultura_mundial

Sevilla, E. G. (2004). Agroecología y agricultura ecológica: hacia una "re" construcción de la soberanía alimentaria. Revistas científicas de la Universidad de Murcia. Recuperado de revistas.Um.es/agroecología/article/download/13

\section{Leyes y otra normativa consultada}

Colombia, Ministerio de Comercio (1993, 15 de enero). "Decreto número 2649 de 1993. Recuperado de http://www.superfinanciera.gov.co/ Normativa/ NormasyReglamentaciones/dec2649-93.pdf

Colombia, Ministerio de Agricultura y Desarrollo Rural (2006, 31 de julio). Resolución número 00187 de 2006. Ministerio Agricultura y Desarrollo 
Rural. Recuperado de http://www.Minagricultura.gov.co/archivos/resolucion187_06.pdf

International Accounting Standards Board (IASB) (2009). Norma Internacional de Contabilidad 38 Activos Intangibles. Recuperado de http://www.mef.gob.pe/ contenidos/conta_publ/con_nor_co/vigentes/nic/38_NIC.pdf

International Accounting Standards Board (IASB) (2010). Marco conceptual para la información financiera. Recuperado de http://contabilidad.bligoo.com/ media/users/3/193927/files/35676/66021300MarcoConceptual.pdf 